ARTIGO

\title{
LA EPISTEMOLOGÍA DE LA EDUCACIÓN FÍSICA COMPARADA: APLICACIONES Y TENDENCIAS
}

\author{
THE EPISTEMOLOGY OF COMPARATIVE PHYSICAL EDUCATION: \\ APPLICATIONS AND TRENDS
}

\author{
A EPISTEMOLOGIA DA EDUCAÇÃO FÍSICA COMPARADA: \\ APLICAÇÕES E TENDÊNCIAS
}

\author{
Adolfo Ramos Lamar \\ Fundação Universidade Regional de Blumenau - Brasil \\ Eduardo Francisco Freyre Roach \\ Universidad Agraria de La Habana - Cuba \\ Fabio Zoboli \\ Universidade Federal de Sergipe - Brasil
}

\begin{abstract}
Resumen: Los conocimientos comparativos se le toma en cuenta en Educación Física a la hora de proyectar, implementar, monitorear, y evaluar políticas, currículos, planes de estudios, clases, e investigaciones. La Epistemología de Educación Física Comparada trata de la validez de esos conocimientos. El objetivo de este artículo es discutir cómo se manifiestan las diversas perspectivas epistemológicas en la producción de conocimientos comparativos para la Educación Física. Se exponen las perspectivas epistemológicas en cuanto a los presupuestos ontológicos y gnoseológicos que sugiere, y sus aplicaciones en el trabajo comparativo. Se concluye que en Educación Física Comparada existen confluencia, complementación, y triangulación entre las perspectivas epistemológicas, así como los métodos y técnicas que promueven, no solo resultan tendencias inevitables, sino también loables. El trabajo sistematiza experiencias de trabajo docente y de investigación de los autores en cuestiones epistemológicas relativas a la Educación Física.
\end{abstract}

Palabras clave: Epistemología; Educación Comparada; Educación Física Comparada.

\begin{abstract}
The Comparative knowledge is considered in Physical Education when planning, implementing, monitoring, and evaluating policies. It also applies to curricula, classes, and research. The Epistemology of Comparative Physical Education deals with the validity of this knowledge. The objective of this article is to discuss how the various epistemological perspectives are manifested in the production of comparative knowledge for Physical Education. The epistemological perspectives regarding the ontological and gnoseological presuppositions that it suggests, and their applications in comparative work, are exposed. It is concluded that in Comparative Physical Education there is a confluence, complementation, and triangulation between the epistemological perspectives, the methods and techniques that they promote. It is not only inevitable but also laudable. The work systematizes experiences of teaching and research work of the authors in epistemological issues related to Physical Education.
\end{abstract}

Keywords: Epistemology; Comparative Education; Comparative Physical Education 
Resumo: O conhecimento comparativo é levado em consideração na Educação Física ao planejar, implementar, monitorar e avaliar políticas, currículos, currículos, aulas e pesquisas. A Epistemologia da Educação Física Comparada trata da validade desses conhecimentos. O objetivo deste artigo é discutir como as diversas perspectivas epistemológicas se manifestam na produção do conhecimento comparativo para a Educação Física. São expostas as perspectivas epistemológicas a respeito dos pressupostos ontológicos e gnoseológicos que sugere e suas aplicações no trabalho comparativo. Conclui-se que na Educação Física Comparada há confluência, complementação e triangulação entre as perspectivas epistemológicas, bem como os métodos e técnicas que promovem, não apenas tendências inevitáveis, mas também louváveis. O trabalho sistematiza experiências de trabalho de ensino e pesquisa dos autores em questões epistemológicas relacionadas à educação física.

Palavras clave: Epistemologia; Educação Comparada; Educação Física Comparada.

\section{Introducción}

¿Quién en su vida no apela a la comparación? Suscitan comparación cosas que a simple vista se ven diferentes, pero se parecen. Hay gran acuerdo en que el comparar es inherente o innata a la mente humana, en fin, una especie de proclividad inconsciente, pero también ex professo.

En la Educación Física, como en otras áreas, existen modos de proceder, métodos, enfoques, técnicas para conocer, comprender saber que algo anda bien o mal, mejor o peor. Un resultado comparativo suele fundamentar la toma de decisiones, y lo susceptible y conveniente de aplicarción total o parcial. Empero, existe la incertidumbre de si una determinada comparación es posible o imposible, en qué medida es atinada o desatinada. La duda pudiera recaer en la supuesta naturaleza propia o particular de las cosas que se comparan. Otras veces en el criterio que arbitrariamente se toma para comparar.

Unos dirían que solo se puede comparar lo comparable, pero otros verían que comparar lo incomparable es un desafío que vale la pena tomar en serio. Pues, tras la apariencia de semejanzas entre determinados elementos se ocultan diferencias (y viceversa) que uno no puede darse el lujo de menospreciar y perder la oportunidad de aprender.

De antaño los filósofos atribuían a la comparación un papel crucial en el conocimiento, ya sea porque se le considera una operación intrínseca del alma o la mente humana, o porque es un proceder indispensable para acceder el conocimiento racionalmente fundamentado. $\mathrm{Al}$ plantear el problema de cuándo podemos considerar que una cosa es conocida o cognoscible (cuestión ontológica), y si el conocimiento que suponemos tenemos sobre ella es posible y valido o confiable (cuestión gnoseológica), ya estamos pisando el terreno de lo que en Filosofía se conoce (salvando las diferencias) como Teoría del Conocimiento, Gnoseología, o 
Epistemología y que se manifiesta en la Educación como un campo relevante de producción y reproducción de conocimientos.

Ahora bien, salta a la vista que los conocimientos en general, e incluyendo los de índole comparativa, son indispensable y recurrentes en Educación. Se les concibe para concebir, implementar, monitorear currículos, cursos, y clases, como de políticas e investigaciones para el desarrollo de la Educación. Permiten conocer mejor las realidades y problemas de la Educación, no solo locales, sino también transculturales o globales (AMARAL, 2015). Este es un punto de partida importante de la Educación Comparada y de los trabajos comparativo en Educación Física (GALAK et al, 2018a; LAMAR, VICENTIN, 2018; TALPE, 2008; QUINTERO, GALAK et al, 2018b; GUTIÉRREZ, DUQUE, 2003).

La Educación tiene que ver crucialmente con conocimientos, incluyendo los de índole comparativa, de ahí que las cuestiones epistemológicas le sean intrínsecas, sean estas referidas al conocimiento en general o al conocimiento comparativo en particular. Siendo la Educación Física una rama de la Educación y la Educación Comparada, la Epistemología de la Educación Física Comparada se encarga de discutir las pretensiones de validez del conocimiento comparativo de la Educación Física.

El presente artículo trata de la Epistemología de la Educación Física Comparada. Expone, analiza, y compara las diversas perspectivas epistemológicas (abordajes, tendencias, enfoques, referenciales, presupuestos, o paradigmas) que concurren hoy en día en la producción de comparaciones para proyectar, implementar, monitorear, y evaluar políticas, currículos, planes de estudios, clases e investigaciones. Es un trabajo teórico, donde se sistematizan experiencias profesionales de los autores en docencia e investigación en Epistemología de la Educación y Educación Física.

Para lograr el objetivo de este estudio, el texto se presenta en cinco secciones. Un primer momento versa sobre la Educación Comparada y Educación Física Comparada para fundamentar lo importante que es la reflexión sobre las cuestiones epistemológicas que plantean. En los apartados dos y tres se exploran las diversas perspectivas epistemológicas que han ido pautando el trabajo en Educación Física Comparada. En la sección cuatro se valora la tendencia a la confluencia, complementación y triangulación de las perspectivas presentada en las partes anteriores. En la quinta y última parte - observaciones finales - hacemos algunos comentarios a modo de síntesis y proposiciones.

\section{La epistemología de la Educación Física comparada}


La Educación Comparada surge "num contexto histórico em que a expansão escolar e a afirmação da ciência se constituíam como pilares fundamentais do progresso, exatamente para poder contribuir para reformas educativas mais fundamentadas" (FERREIRA, 2008, P.125). En este sentido, no es de extrañar que quienes históricamente intentaron establecer pautas o referencias para la Educación Comparada tuvieran que lidiar con pautas epistemológicas para sustentar un método para su investigación. Marc-Antoine Jullien de Paris (1775-1848) fue uno de los pioneros en este empeño. Trató de hacer este movimiento espistemológico por su interés en presentar la Educación Comparada como una ciencia o como un conocimiento intelectualmente sofisticado. En su obra Paris (1967) se perfila muy bien su percepción epistemológica inspirada en el ideal de ciencia del positivismo.

Lógicamente, la cuestión epistemológica sigue replanteándose, una vez que se comienzan a ver los sesgos de esa visión positivista de la Educación Física Comparada, y dada la necesidad de legitimidad los enfoques subversivos o alternativos. Por otro lado,

[...] é natural que a Educação Comparada se veja fortemente condicionada pelos interesses pragmatistas e imediatistas das entidades que dirigem as políticas educativas e que isso suscite algumas reacções dos que recusam aceitar que ela se circunscreva a uma acção meramente técnica e desejam que ela enverede por caminhos mais críticos e reflexivos. (PARIS, 1967, p.126).

La intervención de intereses que pautan el conocimiento en Educación Comparada invita a pensar en qué medida esos intereses sesgan el conocimiento comparativo. Se trata de una cuestión epistemológica crucial que ha estado a la sombra en este campo. Esto implica a la Educación Física, y a la Educación Física Comparada, donde no cabe dudas que hay intereses económicos y políticos en juego. Queda descartada aquí la neutralidad o imparcialidad del conocimiento.

Uno pudiera decir que hay Educación Física Comparada mucho antes de que se estableciera formalmente el campo. Diciéndolo a groso modo, en esta rama se apela a las comparaciones en Educación Física mucho antes de que al estudio comparativo se le otorgara relevancia como enfoque y método, ya no solo a la hora de estudiar los sistemas de Educación Física, sino también cuando se trata de compararlos, sea en el ámbito nacional o internacional.

Hay que destacar que las tendencias de internacionalización, transnacionalización o globalización afectan a la educación, a la Educación Comparada, y a la Educación Física Comparada. Ciertamente: 
económicos, culturales, sociales y políticos transnacionales, así como los procesos transnacionales de regulación educacional, no sólo aquellos promovidos por los organismos internacionales, sino también por los diferentes bloques (Nafta, Unión Europea y, tal vez especialmente Mercosur educativo) enfrentan a los investigadores en política educacional comparada con una serie de desafíos teóricos y metodológicos específicos. Muchos de estos procesos son 'silenciosos', y por eso le cabe a los investigadores identificarlos, porque no por su relativa invisibilidad resultan menos influyentes. (PIOVANI; KRAWCZYK, 2017, p. 835).

En este sentido, cuestiones epistemológicas cruciales comienzan a plantearse en este contexto. Por ejemplo, hasta qué punto son comparables los sistemas nacionales de Educación Física; y si es posible ese conocimiento, dado que el material empírico y las unidades de análisis son sumamente amplias y heterogéneas; y si a este nivel solo se puede comparar lo que se preste a evaluaciones codificadas, cuantificables, y estadísticas, es decir, estandarizadas.

La Educación Física Comparada se presenta como una rama de la Educación Comparada (PADRÓN, 2009, p.11), y por lo tanto su epistemología es subsidiada por la misma. Sin embargo, si bien es cierto que los investigadores en Educación Física Comparada prestan atención a lo que se hacen sus homólogos, también les interesa discutir su modo propio de realizar el trabajo comparativo, que incluye cuestiones relativas a la identidad disciplinar.

Desde finales de 1940, los educadores que se aventuran en Educación Física Comparada "han estado trabajando en sus propias elaboraciones" (KUDLORZ, 1989, p. 651), métodos y enfoques (D’AMICO, YAN HO, s/d, p. 7). Esto se observa muy bien en la masa crítica de trabajos que se exhibe hoy sobre Educación Física Comparada en revistas especializadas, congresos e instituciones nacionales e internacionales. La demanda de lograr mayor coherencia de las investigaciones en Educación Física Comparada, la transferencia, el compartimiento o intercambio de experiencias y colaboración en este campo, justifica que se tome en serio las cuestiones epistemológicas.

Diversas perspectivas, enfoques o paradigmas epistemológicos concurren en Educación Física, lo cual viene suscitando debate, discusiones y controversias en el mundo entero y en Brasil (ALMEIDA et al, 2018; CHAVES, GAMBOA, 2011). Hay quienes se muestran reluctantes respecto a esa diversidad epistemológica, y proponen la imposición de una sola perspectiva válida (monismo), mientras que otros desafían esa actitud (pluralismo), y apuestan a la convivencia, alternancia, confluencia, complementariedad o triangulación entre las mismas. No faltan en Educación Física Comparada trabajos con diferentes perspectivas epistemológicas que se presentan como teorías rivales, como irreconciliables y también se observan actitudes conciliatorias e integracionistas. 
En la literatura de Educación Física suele ocurrir que la comparación no aparece declarada como tema central, ni tampoco como objetivo, enfoque, método o técnica. Pero desde el momento en que se realiza una comparación, hay una epistemología subyacente pautando el trabajo, es decir, hay una idea de las realidades que son comparables (ontología) y de la validez del conocimiento comparativo (gnoseología) que se pretende.

No obstante, un análisis a fondo pondría a esa epistemología al desnudo. Un trabajo comparativono escaparía a un escrutinio o critica desde el punto de vista epistemológico. Diciéndolo de otro modo: hay trabajos que no son netamente comparativos, pero no por ello se le debe eximir de 'vigilancia epistemológica'. Los trabajos netamente comparativos, es decir, cuya intencionalidad es propiamente comparativa de principio a fin, y en todo el proceso, son más proclives de explicitar los presupuestos epistemológicos con los que opera.

\section{La perspectiva epistemológica tradicional en Educación Física comparada}

Pues bien, hay estudios de Educación Física Comparativa (y piénsese también en aquellos donde la comparación no es el enfoque principal) que asumen y promueven la perspectiva epistemológica que aquí llamamos tradicional, convencional o clásica. Puede sospecharse a primera vista, pero no por ello dar por sentado, la presencia de esta perspectiva en los trabajos de Educación Física Comparada donde se procede con realidades, criterios, variables o parámetros, que son susceptibles de cuantificación numérica, calculo y análisis estadístico, y también de estandarización y extrapolación. En tal sentido se comparan, por ejemplo, personas que participan habitual o esporádicamente en actividades físicas (gímnicas, lúdicas, deportivas, fisioterapéuticas o recreativas); actividades de Educación Física, sea dentro o fuera del espacio escolar formal e institucionalizado; recursos o presupuestos destinados a esas actividades; notas de los exámenes escolares, etc.

Supongamos una investigación comparativa que descubre que hay relación (numéricos o estadísticos - cuantitativa) entre el lugar que ocupa un país, una región, o institución, en un determinado ranking, y lo que este país dedica (presupuesto, infraestructura, cursos, horas clases, canchas, eventos, entre otros) para fomentar la educación física y los deportes. Si un país quisiera aprender de otro/s, y quisiera mejorar su desempeño, podría tomar en cuenta los resultados cuantificables que exhibe ese otro país.

Ahora bien, ipreste atención! Cuando se piensa o irreflexivamente se presupone que esta es la única forma legítima de conocer en general y hacer comparaciones legitimas, rigurosas y confiables, estamos en presencia de alguien que adopta un punto de vista 
epistemológico convencional, tradicional o clásico, es decir, que solo se puede hacer comparaciones allí donde es posible aplicar criterios, variables, y parámetros de la índole mencionada, y, además, que el conocimiento obtenido es confiable y replicable. Este presupuesto epistemológico puede estar explícito o implícito en un protocolo o proyecto de investigación, una política, o un currículo, o, simplemente en el discurso de quienes realizan y promueven trabajos en tal sentido.

Esta perspectiva epistemológica tradicional es fácilmente detectable en trabajos y discursos de Educación Física Comparativa que citan a filósofos y establecieron las reglas del juego esenciales y generales para trabajar en esa línea. Son fundamentalmente aquellos filósofos modernos (Descartes y Bacon), positivistas (Comte, Spencer, Mill) y neopositivistas (Círculo de Viena), que en el análisis crítico de Jürgen Habermas (2014), proponían que las ciencias naturales y también las ciencias sociales debían proceder de la misma manera.

Eso significa la pretención de un conocimiento de realidades susceptibles de manejo empírico, es decir, de sistemática y repetida observación, experimento en base a la codificación, y modelación, el establecimiento y operacionalización de variables. Sobre esta base se conciben leyes y regularidades generales y universales (conocimiento nomológico), sobre las cuales se fundamentan decisiones y acciones de índole instrumental, técnica, o mecánica, que, a su vez, propicien ganancias y rendimientos. Y tales acciones han de ser concebidas, dirigidas, monitoreadas, evaluadas y desarrolladas por expertos, e introducidas en los contextos de forma vertical y monológica, es decir, burocrática, o por orden y mando. Es muy usual la adopción consciente o inconsciente de esta perspectiva entre quienes pretenden proyectar decisiones y acciones instrumentales.

Un trabajo de Educación Física Comparativa donde se encuentra subsumida o declarada esta perspectiva, representa francamente esta perspectiva epistemológica tradicional que, en términos de Habermas, se conoce como perspectiva empírico-analítica o positivista. El caso (hipotético) más ilustrativo es cuando se piensa, por ejemplo, que si un sistema determinado de Educación Física registra parámetros cuantitativos favorables, ya previamente definidos, catalogados, y codificados, establecidos, en correspondencia con un determinado ranking, entonces, su experiencia es digna de imitación y extrapolación a contextos similares. Los demás sistemas deben, entonces reformarse a partir de esos criterios.

Desde el punto de vista ontológico esa pretensión empírico-analítica, nomotética, predictiva, e instrumental del conocimiento en Educación Física Comparada, presupone, echar a un lado, borrar o disolver las diferencias o discontinuidades entre las realidades que se comparan. En tal sentido, se tiende a prestarle sobremanera atención y papel a las características 
anatómicas y fisiológicas de las personas, un método único (ejercicios, entrenamiento, instrumentos) para desarrollar sus habilidades o aptitudes físicas, mentales, motrices, o deportivas, así como las correspondientes políticas y diseños curriculares. El modo de concebir la comparación consiste en concebir y aplicar en uno u otro caso una norma o criterio referencial determinado de comparación, diferenciación y jerarquización.

Esto da lugar, por ejemplo, a los procesos de transferencia educativa o extrapolación de aspectos de un sistema de Educación Física hacia otro, con vistas a su modificación o reforma, y basado en un trabajo comparativo con enfoque tradicional sigue el siguiente patrón de acciones pensadas cronológicamente: “(1) se identificó un problema local; (2) se buscaron soluciones en el extranjero y (3) una institución o práctica educativa "probada" (que funcionó o se cree que funcionó) se adaptó al nuevo contexto y luego se implementó" (BEECH, 2006, p.2)

Investigaciones de Educación Física Comparativa, y las acciones y recomendaciones que se conciben en base a sus resultados suelen seguir este orden cronológico. La perspectiva epistemológica tradicional se hace más visible en los discursos y disposiciones institucionales que proyectan políticas de educación física y deportes. $\mathrm{Y}$ en cualquier contexto uno puede encontrar investigaciones que justamente tributan a esas políticas.

Un ejemplo fehaciente fueron las políticas de educación física de la dictadura militar en el Brasil (entre 1954 y 1985), que colocaban el acento en los ejercicios gimnásticos (gimnastismo), los deportes (deportivismo), la salud y la higiene (higienismo), el nacionalismo y el patriotismo (GHIRALDELLI JÚNIOR, 2004).

Hay gran consenso que en muchos lugares esta perspectiva es dominante, pero no hegemónica, expresándose tanto en investigaciones concretas, como en propuestas de modelos de hacer comparaciones (TOOHEY et al, 1981; MORRISON, 1967; BEREDAY, 1964). Sin embargo, el uso de técnicas empíricas (observación y experimento), cuantitativas o estadísticas, no define por sí solo, si quien ejerce el trabajo de Educación Física Comparada asume un enfoque epistemológico empírico-analítico inspirado en el positivismo. Mas bien:

Lo que caracteriza el positivismo es su valoración del método como criterio de validez. Y la intención de encontrar leyes, regularidades, mediante un procedimiento experimental para confirmar hipótesis obtenidas mediante un proceso de abducción. Es la separación de sujeto y objeto, con la consideración de que cualquier tipo de verdad en el sujeto proviene del objeto como adecuación del conocimiento al mundo objetivo mediante un método. Es el apego a las regularidades y el descarte de las singularidades. Es el afán explicativo por considerar un hecho como perteneciente a una clase. Eso es positivismo. No el uso de datos numéricos o su manejo estadístico. (PÉREZ, 2011, p.11). 
Pero esto no sería la única forma de aprender, y se corre el riesgo de la imitación ultranza que no toma en cuenta ni las particulares ni el hecho de que los resultados dependen no solo de esas variables.

\section{La perspectiva epistemológica no-convencional en Educación Física comparada}

En un estudio doctoral comparativo Oreshkina Maria (2007) explora, mediante encuestas, entrevistas, relatos, y reuniones, las experiencias y vivencias de profesores de Educación Física en Sudáfrica, Rusia, y Estados Unidos sobre el trabajo que realizan con estudiantes de bajo rendimiento (Se identificaron similitudes en la estructuración y temática de las distintas experiencias, las cuales propiciaron arribar al concepto del aula (classroom culture), el cual incluye:

[...] (1) enfoque holístico de cada estudiante; (2) crear un lugar seguro para aprender y tomarse el tiempo para establecer una relación con los estudiantes; (3) el enfoque de los maestros en los estudiantes aprendizaje (4) ayudar a los estudiantes a convertirse en individuos independientes y autosuficientes; (5) docentes participación en la vida de los estudiantes; (6) maestros y estudiantes que crecen y cambian juntos; y (7) conocimiento del maestro en sus respectivas disciplinas y alto sentido del maestro. (ORESHKINA MARIA, 2007. p.3).

Otro estudio, cuyo autor define como investigación transcultural en Educación Física (Cross-cultural research in PE), compara una escuela de Alemania y otra de Inglaterra en cuanto a la percepciones e interpretaciones de profesores y alumnos sobre la Educación Física, en correspondencia con sus intenciones, creencias, y el marco legal especifico social, político, escolar y curricular de la Educación Física en esos países (FISHER, 1994). Pues bien, estos son estudios que ilustran muy bien la presencia de una perspectiva epistemológica no tradicional de realizar trabajos en Educación Física Comparada.

En estos estudios se observa, primero, que se trata de describir exhaustivamente, interpretar, y comparar las experiencias o vivencias de las personas de varios países que participaron en la investigación. Se parte de que una persona cuando realiza ejercicios hace deportes, o enseña, instruye o entrena a otros en correr, saltar, hacer fuerza, nadar etc., tiene experiencias, vivencias, percepciones, y creencias, así como expectativas, y la importancia, y el sentido y significado que atribuye a lo que hace. Digamos que aquí cuenta no solo lo que conoce, sus habilidades y practicas o las instrucciones aprendidas, sino también sus emociones, afectos y concepciones sobre sí mismo, los demás, la actividad que realiza, el contexto en que 
esa actividad y la persona se encuentra inmersos. Tales factores experienciales o vivenciales, así como contextuales, juegan un papel no menos importante o crucial que los conocimientos técnicos y las habilidades que aprende para realizar de forma eficaz y eficiente la actividad física, fisioterapéutica, deportiva o recreativa, la eficiencia o la eficacia de la práctica. El sentido de sus vidas, la autoimagen que se construye de sí mismo, o el placer o displacer que reporta la actividad, no son elementos a echar a un lado.

No se pierda de vista que este condicionamiento existencial, emocional, y afectivo, así también como la disposición de intercambios de visiones, experiencias y vivencias, también opera en quienes conciben políticas e investigaciones en función de la educación física.

En segundo lugar, desde el punto de vista de esta perspectiva epistemológica, no se trata de justificar, validar o confirmar un concepto, hipótesis o teoría previamente establecida. $\mathrm{Ni}$ mucho menos se trata de generalizar, explicar y borrar la singularidad de las experiencias. El descubrimiento de un concepto o una categoría se logra, como se ilustra en las citadas investigaciones de Educación Física Comparativa, no por la vía de la generalización de esas experiencias, sino por descripción consistente, sin un concepto previo, e interpretando los testimonios de los profesores participantes para, precisamente, llegar a la elaboración de un concepto que hace coherentes y comprensibles esas experiencias.

Obviamente, son singulares y distintas las realidades, circunstancias o situaciones, en que las personas viven y tienen experiencias y vivencias con sentido y significados. Es por eso que hay quienes se muestran reluctante y se resisten al aprendizaje de las experiencias de otros. $\mathrm{O}$, a lo sumo, confían en el aprendizaje de experiencias de otros que sean medibles o supuestamente objetivas, mas allá de lo que las personas sienten, piensan y desean. Como vivos, esta reluctancia es usual en contextos donde predomina la perspectiva epistemológica tradicional.

En tercer lugar, esta perspectiva sugiere un modo no tradicional de concebir comparaciones que sirvan para proyectar mejoras en los sistemas de Educación Física y los deportes a nivel local, como global. Pero no por ello se cierra la posibilidad de que, a partir del intercambio entre vivencias, se genere un conocimiento aprendido y compartido. Las experiencias descritas y analizadas en comparación ofrecen:

[...] oportunidades para explorar modos alternativos de conciencia y desarrollar ideas y nuevos modos de ser y las posibilidades quizás no estén disponibles en otro lugar en el currículo... (STOLZ, 2013, p. 251). 
Se trata pues no de confirmar nuevas ideas, sino de elaborarlas a partir del material que ofrecen las descripciones e interpretaciones de las experiencias. Una vez que se recolectan, se describan y categorizan sus vivencias, es necesario una retroalimentación constante entre el investigador y los participantes de la investigación que le permita entender de forma cada vez más holística la realidad educativa objeto de estudio. Y esto es precisamente una oportunidad que permite una comparación con más dinamismo y riqueza de matices.

Pero, aún más, es en este proceso de retroalimentación donde radica la forma en que en la investigación fenomenológica es posible auditar los métodos, técnicas, conclusiones y recomendaciones de la investigación, $y$, por ende, considerar que el conocimiento obtenido es confirmado y creíble, su pretensión de validez y veracidad está garantizada por las voces de los participantes y los investigadores, y desde la hora en punto en que las diversas vivencias están representadas y justo valoradas.

Por último, esta perspectiva da relevancia a las técnicas de colección de datos e informaciones sobre las experiencias, sobre todo, a las que utilizan instrumentos como un cuestionario de preguntas, diálogos, encuestas, entrevistas, consulta de expertos, historia de vidas, investigación participativa o colaborativa etc... Tales procedimientos o técnicas cualitativas hoy en día vienen ganando en auge y reconocimiento entre investigadores y metodólogos de la Educación Comparada (SPARKES, 1991; MERRIAM, 1998; MOUSTAKAS, 1994). No obstante, hay que recargar que el hecho de que un investigador apele a instrumentos de investigación cualitativa no significa que se base en presupuestos epistemológicos no tradicionales. En las pesquisas cualitativas se trabaja con múltiples realidades, personas, contextos, y experiencias singulares, por lo que no tiene sentido la replicación o transferencia lineal de los resultados. Pero eso no desautoriza que las evidencias descubiertas de un estudio se puedan repetir en condiciones similares

En ocasiones, por ejemplo, se apela a entrevistas, pero ya las preguntas vienen pautadas por los criterios y variables predefinidos y estandarizados e incluso, reducidos a términos cuantitativos. Hay múltiples elementos que deben considerarse más allá de la elección técnica. (GAMBOA, 2007; GATTI, 2001). Tal y como apuntamos en el caso de la perspectiva tradicional, en lo que concierne a la perspectiva fenomenológica, hay que ver si un trabajo comparativo tributa a un sistema de educación física cuya política precisamente promueve esa epistemología. Debe considerarse también si los trabajos de forma explícita u oculta operan apoyándose en los clásicos que sugieren una epistemología no tradicional.

Fundamentación y sistematización de esta epistemología no convencional se encuentra en los trabajos de filósofos contemporáneos (Dilthey, Husserl, Heidegger, Gadamer, Escuela 
de Frankfort, Habermas, Merleau-Ponty, entre otros), que confluyen en el enfoque crítico de los problemas de la modernidad, la sociedad industrial, y la metodología de la ciencias sociales o humanas, inspirada en la epistemología del positivismo. A partir de Habermas, esta perspectiva se viene conociendo como fenomenológica, hermenéutica, fenomenológico-hermenéutica, o fenomenología transcendental o hermenéutica. Un trabajo de Educación Física Comparativa concebido desde esta perspectiva describe comparativa y sistemáticamente las experiencias subjetivas de las personas, qué es lo que se vivencia y cómo.

Esto presupone el poner entre paréntesis, a un lado, suspenda, o neutralice, las propias actitudes de investigador, sus juicios previos (prejuicios), creencias, suposiciones, sobre las experiencias que se desean describir en profundidad (perspectiva fenomenológica transcendental), interpretar o comprender contextualmente (perspectiva fenomenológica hermenéutica).

Las comparaciones se realizan sobre esos datos. En rigor, o en última instancia, se trata más bien de poner al desnudo o someter esos juicios anticipados a un proceso de reflexión y critica sistemática que permita ver las experiencias comparadas, sin que estos hagan de las suyas y limiten el conocimiento y las experiencias comparadas, sin que estos hagan de las suyas y limiten el conocimiento y el aprendizaje. Por otro lado, esas anticipaciones son una pieza indispensable del trabajo comparativo con pautas o presupuestos fenomenológicos.

Puede resumirse que la ontología de la Educación Física Comparativa, que sugiere esta perspectiva epistemológica, otorga relevancia a la comparación de las visiones, vivencias, creencias, y conceptos de las personas del movimiento y potencialidades de su cuerpo, de las actividades cotidianas que realizan, sus emociones y afectos, así como sus sueños, esperanzas, o frustraciones. Mientras que en lo que atañe a la gnoseología, esta perspectiva le endorsa a tal conocimiento comparativo criterios específicos de validez, confiabilidad y rigor específicos que emanan del proceso de descripción e interpretación sin conceptos previos, sino de los procesos de retroalimentación sistemática entre los investigadores y las personas que dan testimonio, comunican e intercambias sus experiencias.

La perspectiva epistemológica no convencional (en su diversidad de variantes) fundamenta la posibilidad de la comparación válida (verdadera, rigurosa, validable, confiable) de experiencias individuales, más allá de las reglas del juego que invita la perspectiva epistemológica convencional, empírico-analítica o positivista. Digamos que:

[...] cuando el investigador lee y relee el dato, puede decir "ieureka!" porque una de las cosas bonitas de la investigación cualitativa es que todos los días descubrimos, nos sorprendemos de lo que encontramos al releer al otro, al 
aproximarnos a comprender el asunto desde el otro. Hay que recordar que no es una comprensión exclusivamente del otro; el investigador también queda ahí: es un asunto de intersubjetividad. Entonces están los sujetos, el investigador y los dos, que es un tercero. El investigador usa la comparación constante para generar conceptos, no para descubrir similitudes o diferencias significativas. El investigador no se debe quedar en la descripción de las similitudes o diferencias, sino que debe avanzar hacia la generación de conceptos con carácter explicativo. Comparar en rigor permite extraer propiedades y, así, examinar el incidente o las acciones para luego avanzar a identificar las dimensiones, o sea los matices. (YEPES; MOLINA, 2015, p.2.)

Esto implica ir más allá de lo común o compartido entre experiencias, sino en lo que confluyen, más allá de las vivencias particulares que se describen y comparan. Las comparaciones en esta perspectiva epistemológicas no priorizan la búsqueda de representatividad ni tampoco regularidades o generalizaciones, ni mucho menos replicabilidad, más bien, enriquecer y ampliar las perspectivas de análisis y entendimiento de las cosas que se comparan, ya sean por separados o en conjunto. Eso, naturalmente, requiere un trabajo no individual, sino colectivo, donde participan muchas personas; observación persistente, triangulaciones de datos, informaciones, fuentes, y expertos que confirmen que los resultados obtenidos son coherentes, confirmados, y validos (BLANCHARD; MUZÁS, 2016; ERLANDSON et al. 1993; LINCOLN; GUBA, 1985).

\section{La confluencia, complementariedad y triangulación entre las perspectivas epistemológicas en Educación Física comparada}

Recientemente un colectivo de investigadores (COPPOLA; RAIOLA, 2019) que compararon la percepción de los métodos de entrenamiento de profesores de Noruega e Italia, que tomaban como punto de referencia el indicador VO2 de máxima capacidad (VO2max capacity). Una vez descritas, codificadas, calculadas y comparadas las respuestas de los profesores entrevistados, los investigadores concluyeron que por múltiples factores, incluyendo el modo en que trabajaban con ese indicador, los valores favorecían a los profesores noruegos. Ya no solo porque el territorio donde los noruegos realizan entrenamiento es montañoso, sino por los entrenadores noruegos le prestan mayor interés al indicador VO2max capacity, y a las actividades competitivas (más que en entrenamientos), en su trabajo tanto con amateur y con profesionales, tanto en actividades físicas, deportivas, e incluso, recreativas.

En un estudio comparativo sobre los estilos de enseñanzas, los investigadores, definiendo previamente los diferentes estilos, visitan, observan y graban en videos las clases de profesores y a los mismos le hacen entrevistas de lo que piensan sobre el asunto. Asumen de 
antemano que hay tres estilos de enseñanza: estilo comando, estilo práctico y estilo recíproco, los cuales se codifican y sobre esa base se realizan las entrevistas los cálculos de las respuestas en términos estadísticos. Finalmente, los autores concluyen que la pluralidad de maestros prefiere la enseñanza con el estilo de comando que representa el límite inferior de individualización, autonomía y creatividad, ya que el profesor centra su intervención en sus proprias actividades y no en la actividad del alumno (BALI; SOUISSI, 2015).

Por último, citemos someramente un estudio sobre la relación entre los sistemas de política deportiva de élite (entradas y rendimientos) y el éxito en las competiciones internacionales (salidas). Se implementó un modelo conceptual de los factores de política deportiva que conducen al éxito deportivo internacional en un entorno empírico en un estudio piloto con seis naciones. El estudio ha tratado de poner en funcionamiento nueve pilares o conductores clave en los sistemas deportivos de élite en conceptos medibles que se pueden agregar en una puntuación general para cada pilar. Además, de un cuestionario de política deportiva nacional, los atletas, entrenadores y directores de rendimiento también participaron en la recopilación de datos cualitativos y cuantitativos. Aunque los resultados no son concluyentes, (DE BOSSCHER et al., 2009).

Otro estudio (MACHPHAIL, et al, 2018) trata de los mejores patrones de calidad del profesor de Educación Física que ha cursado el programa de formación inicial PETE (PostPrimary Physical Education Teacher Education). Se hace la comparación de esos programas en 26 países europeos, a partir de entrevistas y consultas de expertos de esos programas en esos países. Por medio del uso de la técnica comparativa de codificación abierta se revisaron las presentaciones de los expertos pudiéndose desarrollar categorías de las respuestas dadas por los expertos participantes en la investigación. Todas las presentaciones fueron codificadas, analizadas y trianguladas a través de las grabaciones de las presentaciones, las respectivas sesiones de preguntas y respuestas en las reuniones y borradores de capítulos escritos para una publicación colectiva.

Pues bien, estudios como los citados marcan pautas en la Educación Física Comparativa, en lo que respecta a la tendencia al empleo de metodologías y técnicas mixta, esta hoy en día en auge. Instituciones internacionales como Sociedad Internacional para la Educación Física Comparada y el Deporte (ISCPES) invita a trabajos en esa dirección. Y hay mucho interés en propuesta de modelos de cómo realizar esas investigaciones donde se emplean triangularmente múltiples métodos como las pruebas estandarizadas, entrevistas, encuestas, experimentos de campo, observación participante, etc., lo cual permite "consistencia de los datos y faciliten la generalización de los hallazgos" (OLAFSON, 1991). 


\section{Observaciones finales}

Si usted hace un click en el motor de búsqueda de Google (en cualquier idioma) con entrada de Educación Física Comparada o el método comparativo en Educación Física, le aparecerán muchos trabajos que manifiesta ese interés. Y tal masa bibliográfica critica, así como las instituciones, asociaciones y editoriales que estas detrás de esas producciones, y los esfuerzos por concebir modelos de trabajo técnico o comprensivo (MÉNDEZ; VALERO; CASEY, 2015) en tal sentido, evidencian que la Educación Física Comparada viene constituyéndose en un campo en expansión y que reclama identidad epistemológica específica, sin la necesidad de romper sus lazos con la Educación Comparada.

El interés de comparar personas, actividades, parámetros de desempeño, así como enfoques, metodologías, técnicas, políticas o currículos, se vienen realizando, no solo al interior de los contextos locales, sino también entre los mismos. Ese ensanchamiento del escenario de investigación y los temas de interés resulta un desafío epistemológico, porque las realidades a investigar son más disímiles y complejas. Basta tener en cuenta que en la época en que Platón y sus contemporáneos hicieron comparaciones de la Educación entre pueblos, esos pueblos eran lo que hoy puede ser una comunidad o un barrio. Los países y los sistemas escolares de Educación física hoy en día son más grandes y diversos, y las percepciones sobre cómo concebir la Educación física no son menos diversas.

De ahí, entonces, que se requieren abordajes metodológicos y técnicas múltiples y mixtas para realizan trabajos de Educación Física Comparativa. A su vez, esto es posible si entran en juego diferentes perspectivas epistemológicas.

Como hemos visto, la perspectiva epistemológica tradicional tiene presencia en Educación Física Comparada. Si bien en algunos contextos pudiera descollar como predominante, no por ello pudiera ser hegemónica, pues están ganando auge otras perspectivas, como, por ejemplo, las inspiradas en la fenomenología y la hermenéutica, y otras que no tratamos aquí como las inspiradas en la filosofía critica, el posestructuralismo, Foucault, los estudios poscoloniales y descolonización y el pensamiento complejo.

\section{REFERENCIAS}

ALMEIDA, Felipe Quintão de; GOMES, Ivan Marcelo; SAMPAIO, Amanda; MARINOTTI, Arielli. O corpo como tema da produção do conhecimento: uma análise em cinco periódicos da educação física brasileira. Movimento, Porto Alegre, v. 24, n. 1., p. 133-146, jan./mar.2018. Disponible en: file:///C:/Users/User/Downloads/73680-338118-1-PB.pdf 
AMARAL, Marecelo Parreira do. Tendências, desafios e potenciais da educação internacional e comparada naatualidade. Revista Brasileira de Estudos Pedagógicos, Ago 2015, vol.96, no.243, p.259-281. Disponible en: https://www.scielo.br/scielo.php?pid=S2176$\underline{66812015000200259 \& \text { script}=\text { sci_arttext }}$

BALI, Naila., SOUISSI, Khemais. (2015). Comparative Study of Physical Education Students Teachers Style Interventions Teaching Styles Skill. Creative Education, 6, 100-113. Disponible en: http://dx.doi.org/10.4236/ce.2015.61009

BEECH, Jason. The Theme of Educational Transfer in Comparative Education: a view over time. Research in Comparative and International Education, Vol. 1, No. 1, 2006.

Disponible en: https://journals.sagepub.com/doi/10.2304/rcie.2006.1.1.2

BEREDAY, George. Z. Comparative Method in Education. New York: Holt, Rinehart and Winston Inc, 1964.

BLANCHARD, Mercedes; MUZÁS, María Dolores. La influencia de las narraciones en la construcción de la identidad de las mujeres educadoras en Portugal y España. Revista Práxis Educacional, Vitória da Conquista - Bahia - Brasil, Vol. 12, N. 21, 2016. Disponible en: https://periodicos2.uesb.br/index.php/praxis/article/view/870

CHAVES, Márcia; GAMBOA, Sílvio Sanchéz. A produção de conhecimento em EF/ Ciências do Esporte/ CE - qualidade x quantidade: os desafios de uma experiência concreta. Palestra proferida no IV Fórum de Pós-Graduação em Educação Física do CBCE. Florianópolis, 2011.

COPPOLA, Cecília; RAIOLA, Gaetano. Interest in VO2max capacity: comparing Norwegian and Italian training. Journal of Physical Education and Sport(JPES), Vol.19 (Supplement issue 5), Art 268, pp. 1825-1827, 2019. Disponible

en:https://efsupit.ro/images/stories/october2019/Art\%20268.pdf

D’AMICO, Rosa López de; YAN HO Walter King. Comparative Physical Education And Sport. Sport, Science and Physical Education. Encyclopedia of Life Support Systems (EOLSS), Vol.1. s/d. Disponible en: https://www.eolss.net/Sample-Chapters/C03/E1-12-8905.pdf

DE BOSSCHER, Veerle de; DE KNOP, Peter.; VAN BOTTENBURG, Maateten.; SHIBLI, Simon. A conceptual framework for analysing Sports Policy Factors Leading to international sporting success. European Sport Management Quarterly, 6 (2), 185-215, 2006.

Disponible en:

https://www.researchgate.net/publication/46681518_A_Conceptual_Framework_for_Analysi ng_Sports_Policy_Factors_Leading_to_International_Sporting_Success

ERLANDSON, David; HARRIS, Edward; SKIPPER, Barbara; ALLEN, Steve. Doing naturalistic inquiry: a guide to methods. Newbury Park, CA: Sage Publications, Inc. 1993.

FERREIRA, Antonio Gomes. O sentido da Educação Comparada: Umacompreensão sobre a construção de umaidentidade. Revista Educação, Porto Alegre, v. 31, n. 2, p. 124-138, maio/ago. 2008. Disponible en:

https://revistaseletronicas.pucrs.br/ojs/index.php/faced/article/view/2764 
FISHER Richard John. Physical Education in England and Germany: A comparative tale of two schools of two schools. Thesis submitted for the Degree of Doctor of Philosophy Department of Linguistic and International Studies University of Surrey, 1994.

GALAK, Eduardo; GOMES, Ivan Marcelo; ALMEIDA, Felipe Quintão de; ZOBOLI, Fabio. O gênero nos estudos sobre corpo em revistas do campo da educação física da Argentina e do Brasil. Revista Filosofia e Educação, Campinas, SP, v.10, n.3, p. 598-617, set./dez. 2018a. Disponible en: https://periodicos.sbu.unicamp.br/ojs/index.php/rfe/article/view/8653777

GALAK, Eduardo; ZOBOLI, Fabio; GOMES, Ivan Marcelo; ALMEIDA, Felipe Quintão de. O corpo no campo acadêmico da Educação Física na Argentina e no Brasil: crítica e renovação da disciplina. Revista da ALESDE, Curitiba, v. 9, n. 2, p. 79-90, setembro 2018b. Disponible en: https://revistas.ufpr.br/alesde/article/view/61266

GAMBOA, SílvioSanchéz. A pesquisa em educação: métodos e epistemologias. Chapecó: Argos, 2007.

GATTI, Bernardete A. Implicações e perspectivas da pesquisa educacional no Brasil contemporâneo. Cadernos de pesquisa, n. 113, p. 65-81, julho, 2001.

GHIRALDELLI Júnnior, Paulo. Educação Física progressista: a pedagogia crítico-social dos conteúdos e a Educação Física brasileira. 9 ed. São Paulo: Loyola, 2004

HABERMAS, Jürgen. Conhecimento e interesse. Editora UNESP, 2014.

KUDLORZ, Pawel. Comparative physical education: An international scientific approach. International Review of Education 35(1): 65-72, 1989. Disponible en: https://link.springer.com/article/10.1007/BF00597684

LAMAR, Adolfo Ramos; VICENTIN, Taiane. Epistemologia e educação comparada na América Latina e no Caribe: algumasconcepções. Revista Filosofia e Educação, Campinas, SP, v.10, n.3, p.618-634, set./dez. 2018. Disponible en: https://periodicos.sbu.unicamp.br/ojs/index.php/rfe/article/view/8653809

LINCOLN, Yvona; Guba, Egon. (1985). Naturalistic Inquiry. Newbury Park, CA: Sage Publications. 1985.

MACPHAIL, Ann; BULCA, Yesim; CARRARO, Atillio; AVSAR, Züleyha. Initial PostPrimary Physical Education Teacher Education: A Comparative Exploration across European Countries. International Journal of Sports and Physical Education (IJSPE), vol 4, no.4, 2018, pp. 15-22. Disponible en: https://www.arcjournals.org/pdfs/ijspe/v4-i4/3.pdf

MÉNDEZ, Antonio; VALERO, Alfonso; CASEY, Ashley. What are we being told about how to teach games? A threedimensional analysis of comparative research into different instructional studies in Physical Education and School Sports. Revista Internacional de Ciencias del Deporte. 18(6), 37-56, 2010. http://www.cafyd.com/REVISTA/01803.pdf

MERRIAM, Sharan. Qualitative Research and Case Study Applications in Education. San Francisco: Jossey-Bass, 1998. 
MORRISON, Donald Hugh. Rationale for the development of of comparative physical education. M. Ed. thesis, University of Alberta, 1967.

MOUSTAKAS, Clark. Phenomenological research methods. Sage Publications, Inc.1994

OLAFSON, Guy. Triangulation in comparative research: mixing qualitative and quantitative methods. In: Standeven, J., Hardman, K. and Fisher, R. (Eds), (1991), pp. 3944, 1991.

ORESHKINA, Maria J., "Teachers' Experience of Working With Underachieving Students: A Comparative Phenomenological Study of Teachers in South Africa, Russia, and the United States. " PhD diss., University of Tennessee, 2007. Disponible en: https://trace.tennessee.edu/utk_graddiss/256

PADRÓN, Violeta Rodríguez. La Educación Física Comparada: Aproximaciones a su comprensión como área de la Educación Comparada. PODIUM - Revista de Ciencia y Tecnología en la Cultura Física, [S.1.], v. 4, n. 1, p. 39-53, mar. 2009. ISSN 1996-2452. Disponible en: http://podium.upr.edu.cu/index.php/podium/article/view/56

PARIS, Marc-Antoine Jullien. de. Esboço de uma obra sobre a Pedagogia Comparada. Tradução Joaquim Ferreira Gomes. Coimbra. 1967.

PÉREZ, Darío Alberto Ángel. La hermenéutica y los métodos de investigación en ciencias sociales. Estud.filos, $n^{\circ} 44$, Diciembre, Universidad de Antioquia, pp. 9-37, 2011. Disponible en: http://www.scielo.org.co/pdf/ef/n44/n44a02.pdf

PIOVANI, Juan Ignacio; KRAWCZYK, Nora. Los Estudios Comparativos: algunas notas históricas, epistemológicas y metodológicas. Educação \& Realidade, Porto Alegre, v. 42, n. 3, p. 821-840, jul./sept. 2017. Disponible en: https://www.scielo.br/scielo.php?script=sci_arttext\&pid=S2175-62362017000300821

QUINTERO, Sandra Pulido; GUTIÉRREZ, Carmem Emilia García; DUQUE, León Jaime. Influencia de las ideas modernas en la educación del cuerpo en el ámbito escolar de los discursos de la Educación Física del siglo XIX en Medellín. Efdeportes Revista Digital, Buenos Aires, Año 9 - No 64 - Septiembre de 2003. Disponible en:https://www.efdeportes.com/efd64/ideas.htm

SPARKES, Anton. Exploring the subjective dimension of curriculum change. In: ARMSTRONG, N.; SPARKES, A. (Ed), Issues in Physical Education, London, Cassell Education, pp. 20-35, 1991.

STOL, Steven A. Phenomenology and Physical Education, Educational Philosophy and Theory. Journal Educational Philosophy and Theory, Vol. 45, n.9, 2013. Disponible en: https://www.tandfonline.com/doi/abs/10.1080/00131857.2013.785355

TALPE, Margarite Monbaliu. El currículum de educación física en Bélgica y España: estudio comparado de las prescripciones legales en el primer nivel de concreción: ¿es contraproducente tanta concreción? Revista Española de Educación Comparada, 14, p. 307-343, 2008. Disponible en: 
file:///C:/Users/User/Desktop/TEXTOS\%20EM\%20CONSTRU\%C3\%87\%C3\%830/EF\%20 COMPARADA/El_curriculum_de_Educacion_Fisica_en_Belgica_y_Espa\%C3\%B1a.pdf

TOOHEY, Dick.; TOOHEY, Marc.; HOWELL, Matheus.; HOWELL, Rill. (1981),

Methodology in Comparative Physical Education and Sport, Champaign.Illinois: Stipes

Publishing Co. 1981.

YEPES Carlos; MOLINA Diana. La comparación en el análisis de la investigación cualitativa con teoría fundada. Revista Faculdad Nacional Salud Pública 2015; 33 (supl 1). Disponible en: https://www.redalyc.org/pdf/120/12042407023.pdf

\section{SOBRE OS AUTORES:}

\section{Adolfo Ramos Lamar}

Pós-doutor em Educação pela Universidade de São Paulo - USP. Doutor em Educação pela Universidade de Campinas - UNICAMP. Professor do Programa de Pós-graduação da FundaçãoUniversidade Regional de Blumenau - PPGED/FURB - Brasil. Membro do Grupo de Pesquisa Educogitans. E-mail: ajemabra@yahoo.com.br

(iD https://orcid.org/0000-0003-1164-1172

\section{Eduardo Francisco Freyre Roach}

Doutor em Ciências Filosóficas pela Universidade Estatal de Moscou - Rússia. Universidad Agraria de la Habana - Cuba.Posdoutoral FE UNICAMP, ExProfessor Universidad Agraria de la Habana (UNAH), Cuba.Master en BuddhistStudies (Universityof Hong Kong). Membro do Grupo de Pesquisa Educogitans. E-mail: freyre.roach2016@gmail.com

(iD) https://orcid.org/0000-0002-2267-1564

\section{Fabio Zoboli}

Pós-doutor em Educação do corpo pela Universidad Nacional de La Plata - UNLP/ Argentina. Doutor em Educação pela Universidade Federal da Bahia - UFBA. Professor do Programa de Pós-graduação em Educação da Universidade Federal de Sergipe - UFS/Brasil. Membro do Grupo de Pesquisa "Corpo e Política". E-mail: zobolito@gmail.com

iD http://orcid.org/0000-0001-5520-5773 